\title{
Characteristic Analysis of Abnormal-Weakening Summer Precipitation in Tianjin under the Influence of Bohai Sea High
}

\author{
Changyi $\mathrm{Xu}^{1}$ and Yan Wang ${ }^{2, *}$ \\ ${ }^{1}$ Binhai New Area Meteorological Office of Tianjin, Tianjin 300457, China \\ ${ }^{2}$ Tianjin Weather Modification Office, Tianjin 300074, China
}

\begin{abstract}
To investigate the characteristics of Bohai High, the daily precipitation data of Beijng-Tianjin region during June to September from 2006 to 2015, national basic automatic weather stations data of 5 minute, NCEP/NCAR $1^{\circ} \times 1^{\circ}$ reanalysis data as well as CINRAD/SA data of Tianjin, from the definition of and the distribution characteristics of precipitation in Beijng-Tianjin region, the concept of regional precipitation reduction in summer in the context of Bohai High was given. This was done by classifying large-scale synoptic circulation and synthetic diagnosis method. The results show that: (1) Bohai High has the most significant effect of abnormal weaken precipitation in the eastern of Tianjin, while has a relatively mild effect in the northern of Tianjin. (2) According to the characteristics of large-scale circulation situation, the synoptic processes over heavy rain are classified into three patterns: upper-level trough pattern (I), cold vortex pattern (II) and the interaction between subtropical high and western trough (III). Except that water vapor come from the Bohai Sea and the Yellow Sea for Type II, for the other two patterns the water vapor were related to remote supplies from the South China Sea and the East China Sea. The Bohai High of Type Iand III were thick warm negative vorticity system whereas the Type II was a shallow negative vorticity system. (3) The abnormally weakened of Bohai High were related to their depths and duration, the deeper and the longer duration of Bohai High, the stronger weakening effect on the precipitation in Tianjin.
\end{abstract}

\section{Introduction}

Bohai Sea high (BSH) is a meso-scale anticyclone circulation system that appears in the Bohai Rim in summer ${ }^{[1]}$. Compared with other weather systems (subtropical high ${ }^{[2-11]}(\mathrm{SH})$ and North China high ${ }^{[12-13]}$ ), the intensity and range of BSH are relatively weak, so it is often neglected by researchers. However, when the BSH occurs, the location of summer precipitation in Tianjin is often shifted, the rain belt is broken or the precipitation is weakened by affecting the velocity, direction or intensity of westerlies or the southern branch trough, leading to the abnormal-weakening precipitation. Forecasters often fail to grasp the development trend and the displacement boundary of such precipitation weather processes, resulting in obvious forecast errors.

At present, there are many studies on the influence of westerlies or southern branch trough on summer precipitation in Tianjin [14-17], and the corresponding forecast accuracy is quite high. But the forecasts of heavy rainfall in summer in Tianjin under the influence of $\mathrm{BSH}$ are often false. Therefore, it is of great significance to understand the influence and role of BSH on summer precipitation in Tianjin, for establishing a forecast idea and reducing the false rate. Although previous studies indicate preliminary understanding of the formation mechanism of BSH, synoptic-scale circulation characteristic analysis based on long time series and meso-scale characteristic analysis based on individual cases are both difficult to reveal the effect of the BSH on abnormal-weakening summer precipitation in Tianjin. In this study, the synthetic diagnostic analysis method is used to better understand the difference characteristics of abnormal-weakening of summer precipitation in different regions of Tianjin under the background of synoptic scale systems.

\section{Standard, data and methods}

\subsection{The standard of BSH}

The BSH in this paper is mainly based on the definition by Hou Shumei et al. ${ }^{[1]}$. It is stipulated that at $850 \mathrm{hPa}$ (700 hPa, $925 \mathrm{hPa}$, or all three layers), if the wind filed along seven stations (Dalian, Jinzhou, Chifeng, Beijing, Jinan, Qingdao and Chengshantou) around the Bohai Sea can form a closed high-pressure circulation, or the wind filed along five stations (Jinzhou, Chifeng, Beijing, Jinan and Qingdao) can form a anticyclone circulation, it can be defined as a BSH.

* Corresponding author: swallownx@163.com 


\subsection{Data and methods}

NCEP/NCAR 6-hour reanalysis data with an interval of $1^{\circ} \times 1^{\circ}$, conventional meteorological observation data, Sband Doppler weather radar data and automatic station data with an interval of 5 minutes in Tianjin are used in this paper. According to the definition of $\mathrm{BSH}$, the upperlevel data from Micaps are used to select the daily wind and pressure fields at $925 \mathrm{hPa}, 850 \mathrm{hPa}$ and $700 \mathrm{hPa}$ from June to September during 2006-2015. The day with clockwise wind field and higher pressure in the Bohai Sea is defined as a BSH day. The selected case must satisfy the following three criteria at the same time: (1) the occurrence date is a BSH day. (2) The 24-h average accumulated precipitation in Beijing plain area is more than or equal to $25 \mathrm{~mm}$. (3) The 24-h average accumulated precipitation in any or all areas of the northern, central and southern and eastern Tianjin is less than $25 \mathrm{~mm}$, which is defined as the northern, southern or eastern weakening pattern, respectively.

\section{Selection process and its characteristics}

Based on the atmospheric circulation basic elements of the same-pattern heavy rainfall days, the average fields (i.e. composite fields) are calculated. According to the characteristics of large-scale circulation situation, the synoptic processes over heavy rain (more than or equal to $25 \mathrm{~mm}$ ) are classified into three patterns: (1) upper-level trough pattern (pattern I, including straight westerly wind pattern), accounting for $62.5 \%$ of total. Its main feature is that the westerly wind dominates the middle and high latitudes at $500 \mathrm{hPa}$, and the $\mathrm{SH}$ distributes like an eastwest band. (2) Cold vortex pattern (pattern II, including the direct influence pattern of the cold vortex in the southeast quadrant and the northwest airflow pattern behind the cold vortex over Northeast China), accounts for $25 \%$. Its main feature is that the upper level in Inner Mongolia or Northeast China is controlled by deep cold vortex. (3) The interaction pattern (pattern III) of SH and the westerly trough (or low vortex) accounts for $12.5 \%$. Its main feature is that the circulation at $500 \mathrm{hPa}$ presents a great meridionality and the $\mathrm{SH}$ distributes in a shape of block. The 588dagpm isoline extends from the south of $30^{\circ} \mathrm{N}$ to $40^{\circ} \mathrm{N}$ nearby. The strong southerly airflow is accompanied by the low-level jet and the westerly trough is deep. It can be seen from eight abnormal-weakening precipitation cases in summer over Tianjin that the BSH has the most significant effect on the abnormal-weakening precipitation in the eastern of Tianjin, while its effect on the northern Tianjin is relatively weak.

Table 1. Occurrence dates and circulation patterns of weakening precipitation over Tianjin under the influence of BSH from June to September during 2006-2015.

\begin{tabular}{|c|c|c|}
\hline Date & Type & $\begin{array}{c}500 \mathrm{hPa} \\
\text { circulation } \\
\text { pattern }\end{array}$ \\
\hline 20070627 & weakening pattern & $\mathrm{I}$ \\
\hline
\end{tabular}

\begin{tabular}{|c|c|c|}
\hline 20080616 & weakening pattern & I \\
\hline 20100709 & eastern weakening pattern & I \\
\hline 20120624 & $\begin{array}{c}\text { southern, eastern } \\
\text { weakening pattern }\end{array}$ & II \\
\hline 20120901 & $\begin{array}{c}\text { southern, eastern } \\
\text { weakening pattern }\end{array}$ & III \\
\hline 20130707 & weakening pattern & I \\
\hline 20140616 & weakening pattern & II \\
\hline 20140901 & $\begin{array}{c}\text { southern, eastern } \\
\text { weakening pattern }\end{array}$ & I \\
\hline
\end{tabular}

\section{Characteristic Analysis of synoptic- scale systems}

\subsection{Background characteristics of the synoptic- scale circulation with a pattern of upper-level trough}

The circulation features with pattern I under the background of BSH from June to September during 20062015 are compositely analyzed. The upper-level trough at $500 \mathrm{hPa}$ is located near $110^{\circ} \mathrm{E}$, and the temperature trough is ahead of it. And Tianjin is under the control of a weak high pressure ridge, which blocks systems coming from west, and the average wind speed is only $4 \mathrm{~m} \cdot \mathrm{s}^{-1}$. The water vapors from the southwest and southeast at $850 \mathrm{hPa}$ are only transported to the Shandong Peninsula, and Tianjin is in the divergent region of water vapor fluxes. Seen from the wind shear distribution of $0-2 \mathrm{~km}$, the northeastern Beijing and the northern Tianjin are in highvalue areas of vertical wind shear, while the southeastern Tianjin and the Bohai Sea are in low-value areas. It can be seen from the vertical profile of composite wind fields along $39^{\circ} \mathrm{N}$ that the anticyclone circulation at $118-123^{\circ}$ E extends to about $300 \mathrm{hPa}$. That is, the $\mathrm{BSH}$ with pattern I extends relatively high. The southeast airflow exists over the eastern Tianjin and the western Bohai Sea in the lower troposphere of $700 \mathrm{hPa}$, and the southwest airflow is in the middle layer. There is an obvious northwest airflow at 200 $\mathrm{hPa}$, with the average wind speed of about $40 \mathrm{~m} \cdot \mathrm{s}^{-1}$. It can be concluded that the wind is rotating clockwise with height in the whole layer and the warm advection is in charge under the background of BSH.

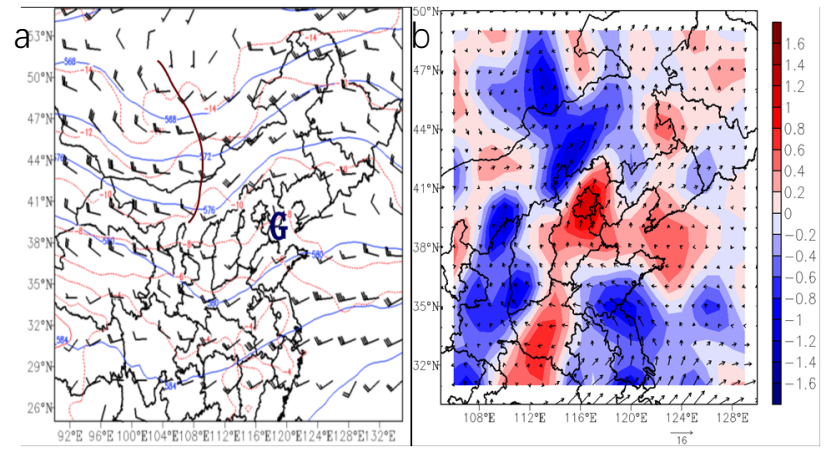



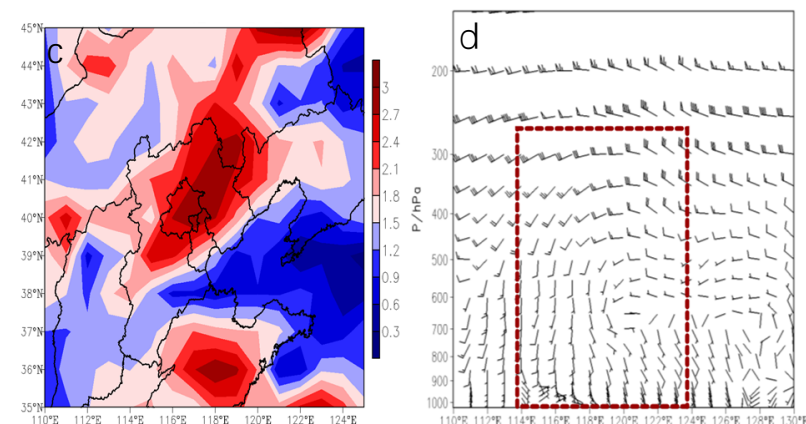

Fig.1. The pattern of upper-level trough synthesis field (a) 500 $\mathrm{hPa}$ geopotential height fields(soild, unit: dagpm), isotherm (dashed, unit: ${ }^{\circ} \mathrm{C}$ ) and wind (b) $850 \mathrm{hPa}$ water vapor flux divergence (shaded, unit: $\left.10^{-5} \mathrm{~g} \cdot\left(\mathrm{hPa} \cdot \mathrm{m}^{2} \cdot \mathrm{s}\right)^{-1}\right)$ and water vapor flux vector (unit: $\mathrm{g} \cdot(\mathrm{hPa} \cdot \mathrm{m} \cdot \mathrm{s})^{-1}$ ) (c) the $0-2 \mathrm{~km}$ wind shear(units: $\left.10^{-3} \mathrm{~s}^{-1}\right)(\mathrm{d})$ the cross section of composite wind fields along $39^{\circ} \mathrm{N}$

\subsection{Background characteristics of the synoptic- scale circulation with a pattern of cold vortex}

Seen from the composite $500 \mathrm{hPa}$ geopotential height fields of pattern II, the cold vortex is located near Lake Baikal, and the upper-level trough is located near $110^{\circ} \mathrm{E}$, and Tianjin is controlled by the southwest airflow in front of the upper-level trough with the average wind speed of only $6-8 \mathrm{~m} \bullet \mathrm{s}^{-1}$, which is stronger than that of pattern I. The area around the Bohai Sea is controlled by a high pressure ridge. The water vapors at $850 \mathrm{hPa}$ by the southwest and southeast wind extend from the southeastern China, the East China Sea and the South China Sea to the Bohai Sea. Tianjin is located in the divergence zone of water vapor fluxes, with the water vapor flux divergence reaching $1.5 \cdot 10^{-5} \mathrm{~g} \bullet\left(\mathrm{hPa}^{2} \mathrm{~m}^{2} \bullet \mathrm{s}\right)^{-1}$. The wind shear distribution of 0 $2 \mathrm{~km}$ shows that the high-value area is located in Beijing, while the low-value area is located in the southeastern Tianjin and Bohai Sea, and the vertical wind shear gradient at low level is great in the northern and southern Tianjin. It can be seen from the vertical profile of composite wind fields along $39^{\circ} \mathrm{N}$ that the anticyclone circulation at $118-123^{\circ} \mathrm{E}$ extends only from $900 \mathrm{hPa}$ to about $500 \mathrm{hPa}$, whose thickness is much thinner than that of pattern I.

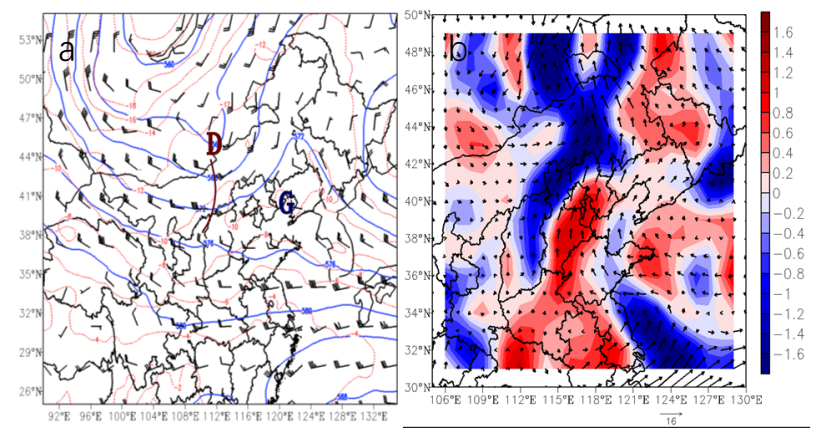

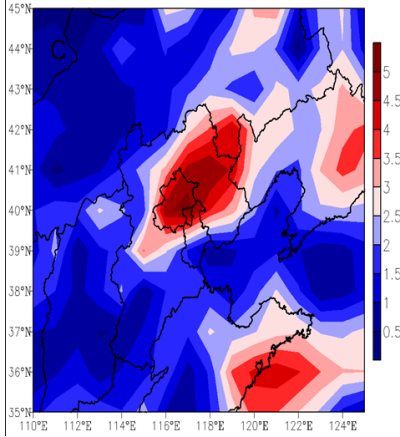

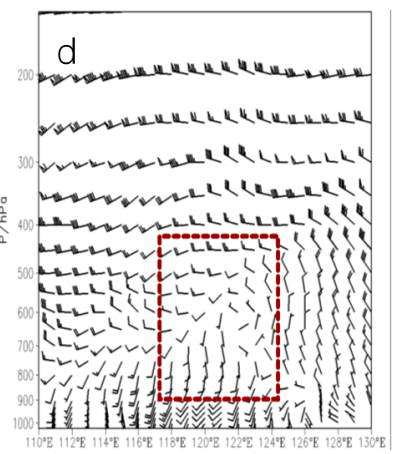

Fig.2. As in Fig.1, but for the pattern of cold vortex

\subsection{Background characteristics of the synoptic- scale circulation with a pattern of $\mathrm{SH}$}

Seen from the composite 500-hPa geopotential height fields of pattern III, the middle and low latitudes of $95 \sim 105^{\circ} \mathrm{E}$ are characterized by a ladder-trough structure and the $\mathrm{SH}$ is distributed in the shape of block. The 588 dagpm isoline extends to the vicinity of $35^{\circ} \mathrm{N}$. The southwest wind at $850 \mathrm{hPa}$ transports the water vapor from the southwestern China to Beijing and Hebei. While the anticyclone circulation around the Bohai Sea blocks the southwest water vapor from transporting to Tianjin. Tianjin is located in the divergent region of water vapor fluxes. The anticyclone circulation near $117-123^{\circ} \mathrm{E}$ extends to $350 \mathrm{hPa}$ vertically, whose thickness is slightly thinner than that of pattern I. Different from pattern I and pattern II, the high-value area of $0-6 \mathrm{~km}$ vertical wind shear is located in the northwestern Beijing for pattern III, the vertical wind shear value of the whole layer over Tianjin area is lower, while the vertical wind shear value in the lower layer of the three patterns are all lower. It can be seen that the vertical wind shear in the lower layer is not the reason for pattern III to weaken the precipitation in Tianjin.
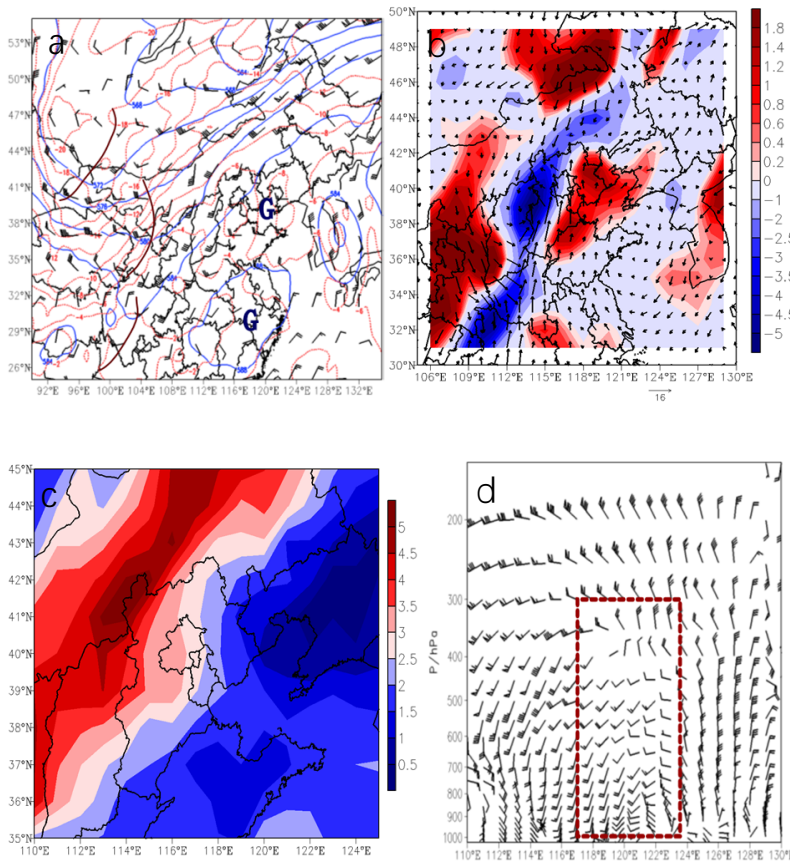

Fig. 3. As in Fig.1, but for the pattern of SH 


\section{Conclusions}

Based on multi-source data and through weather classification of abnormal-weakening summer precipitation cases in Tianjin under the influence of $\mathrm{BSH}$, the effects of $\mathrm{BSH}$ are discussed by analyzing the characteristic differences of BSH under three weather systems. Conclusions are drawn as follows.

(1) The effect of BSH on abnormal-weakening summer precipitation is the most significant in the eastern part of Tianjin, while its effect is relatively weak in the northern part of Tianjin.

(2) Under the background of BSH, the main weather systems bringing abnormal-weakening summer precipitation are divided into three patterns, namely, the upper-level trough pattern (pattern I), the upper-level cold vortex pattern (pattern II) and the SH pattern (pattern III). The water vapor in pattern I and pattern II is mainly related to the long-distance transport from South China, the East China Sea and South China Sea. The water vapor in pattern III mainly comes from the southwestern China, with the most precipitable water in the whole layer. The BSH is a deep warm high-pressure with negative vorticity under the background of upper-level trough and $\mathrm{SH}$, while it is a shallow high-pressure with negative vorticity under the background of cold vortex.

\section{Acknowledgement}

Supported by the National Science Foundation of China (41675046, 40975026)

\section{References}

1. Hou Shumei, Sun Zhongxin, Zhang Chengwang. Analysis of formation mechanism of Bohai High. Shandong Meteor, 27 ,3 (2007)

2. Duan Liyao, Rong Yanshu, Liang Pingde. Effect of west pacific subtropical high on summer precipitation in North China. Meteor Sci \& Tec, 36,3 (2008)

3. He Qunying, Sun Yixin, Liu Yiwei, et al. Tentative analysis of a continuous local heavy rainfall event over Tianjin on 25 July 2012. Torrential Rain and Disasters, 32,3 (2012)

4. Wang Jing, Lv Jiangjin. Analyses of the atmospheric circulation characteristics over Tianjin area in the years of anomalous precipitation in rainy season. Meteor Mon, 37,8 (2011)

5. Sun Weiguo, Cheng Bingyan, Guo Qu. Influence of the subtropical high over western pacific on the difference between precipitation and evaporation in North China. Plateau Meteor, 28,5 (2009)

6. Tan Guirong, Sun Zhaobo. Relationship of the subtropical high and summertime floods/droughts over North China. J Tropical Meteor, 20,2 (2004)

7. Hao Lisheng, Min Jinzhong, Ding Yihui. Analysis of precipitation events changes and causes for rainstorm events reduction in North China. Chinese J. Geophys,54,5 (2011)
8. Hou Shumei, Guo Junjian, Zhang Lei, et al. Analysis of dynamic and thermal field structure characteristics on rainstorm area in interaction process between westerly trough and subtropical high. Meteor Mon, 43,2 (2017)

9. Jin Ronghua, Meng Jiachuan, Pei Haiying. A comparison of three dimension structure of subtropical high for two regional heavy rainfall events. Meteor Mon, 33,4(2007)

10. Liu Huanzhu, Wang Weiguo, Shao Mingxuan, et al. A case study of the influence of the western pacific subtropical high on the torrential rainfall in Beijng area. Chinese Journal of Atmosperic Sciences, 31,4 (2007)

11. Zhou Mingsheng, Duan Liyao, Zhou Liangdan. Statistical analysis of Tianjin precipitation in midsummer and North China High in early summer. Meteor Sci \& Tec,,32,6 (2004)

12. Liang Pingde, Duan Liyao, Zhou Mingsheng, et al. Circulation patterns for mid-summer drought/flood in North China and their early-summer precursers. Acta Meteor Sinica,64,3 (2006)

13. Zheng Lina, Shi Shaoying, Hou Shumei. Contribution of the Bohai Sea's special topography to cold vortex snow event. Meteor Mon, 29,1 (2003)

14. Cao Shuyi. The observations of the Huanghai sea, Bohai sea and the sea of Japan highs and the heavy rain in North China in summer. J Appl Meteor Sci, 1,2 (1990)

15. Cao Shuyi, Qiu Yongyan. The total energy variation during the Northward after Typhoon 9608 landfall over China and the maintenance of Bohai Japanese sea high. Meteor Mon, 24,6 (1998)

16. Qiu Yongyan. Weather patterns and interannual variations of typhoon torrential rain during midsummer in North China. Meteor Mon, 23,7 (1997)

17. Zhang Nan, He Qunying, Liu Yiwei, et al. Multi-scale comparative analysis of two torrential rain events in Tianjin on the subtropical high. Torrential Rain and Disasters, 33,4 (2014) 